\title{
AVALIAÇÃO DO PROCESSO ENSINO- APRENDIZAGEM NO AMBIENTE DE SIMULAÇÃO REALÍSTICA NA GRADUAÇÃO EM ENFERMAGEM
}

Fabiola Lopes Rodrigues ${ }^{1}$ Luciana Melo de Moura² Lara Mabelle Milfont Boeckmann ${ }^{3}$ Manuela Costa Melo ${ }^{1}$

Fabiana Cláudia de Vasconcelos França ${ }^{4}$ Geisa SantAna ${ }^{1}$ http://orcid.org/0000-0002-8646-6316

http://orcid.org/0000-0002-4514-2171

http://orcid.org/0000-0002-1213-559X

http://orcid.org/0000-0002-2018-1801

http://orcid.org/0000-0002-2383-4900

http://orcid.org/0000-0001-8914-4972

Objetivo: Avaliar o processo ensino-aprendizagem no ambiente de simulação realística na percepção de estudantes de graduação em enfermagem. Método: Estudo transversal descritivo com 59 estudantes. Foram aplicadas duas escalas: Experiência com o debriefing e design de Simulação. Dados analisados com Software Statistical Package for the Social Sciences, versão 24.0 , The R Project for Statistical Computing versão 3.3 e Office Excel 2007. Empregaram-se Alpha de Cronbach e teste Qui-quadrado considerando p<0,05. Resultados: para as duas escalas, demostrou-se concordância entre 62\% e 93\% correspondentes ao somatório dos parâmetros: concordo com a afirmação e concordo com afirmação totalmente. A confiabilidade interna da escala de experiência com debriefing foi 0.972 e da escala do design de simulação 0.964. Associando-se dimensões da escala de debriefing, obtiveram-se p-valores significativos de (0-0,0006) e para a escala de design, p-valores (0-0,0050). Conclusões: consolidou-se a simulação como estratégia de ensino-aprendizagem evidenciada pela avaliação satisfatória dos estudantes.

Descritores: Simulação, Educação em Enfermagem, Estudantes de Enfermagem, Simulação de Paciente, Desempenho de Papéis.

\section{EVALUATION OF THE TEACHING-LEARNING PROCESS IN A REALISTIC SIMULATION ENVIRONMENT FOR UNDERGRADUATE NURSING}

Objective: To evaluate the teaching-learning process in a realistic simulation environment according to the perception of undergraduate nursing students. Method: Descriptive cross-sectional study with 59 students. Two scales were applied: Debriefing Experience Scale and Simulation Design Scale. Data were analyzed using the Software Statistical Package for Social Sciences, version 24.0, The R Project for Statistical Computing version 3.3, and Microsoft Office Excel 2007. Cronbach's Alpha and Chi-square test were used considering $p<0.05$. Results: Both scales showed agreement between $62 \%$ and $93 \%$, corresponding to the sum of the parameters: I agree with the statement and I totally agree with the statement. The internal reliability was 0.972 for the debriefing experience scale and 0.964 for the simulation design scale. The association between the dimensions of the debriefing scale showed significant $p$-values $(0-0,0006)$; the simulation design scale showed $p$-values of $(0-0,0050)$. Conclusion: Simulation was evidenced as a teaching-learning strategy, as demonstrated by the satisfactory evaluation of the students.

Descriptors: Simulation, Education Nursing, Students Nursing, Patient Simulation, Role Playing.

\section{EVALUACIÓN DEL PROCESO DE ENSEÑANZA Y APRENDIZAJE EN SIMULACIÓN REALISTA EN ESTUDIANTES DE ENFERMERÍA}

Objetivo: Evaluar el proceso de enseñanza y aprendizaje en un ambiente de simulación realística en la percepción de los estudiantes de enfermería. Método: estudio descriptivo de corte transversal con 59 estudiantes. Se aplicaron dos escalas: experiencia con el informe y diseño de simulación. Las pruebas alfa y chi-cuadrado de Cronbach se utilizaron considerando $p<0.05$. Resultados: Dos escalas mostraron un acuerdo entre $62 \%$ y $93 \%$ correspondiente a la suma de los parámetros: estoy de acuerdo con la declaración y estoy totalmente de acuerdo con la declaración. La confiabilidad interna de la escala de experiencia informativa 0.972 y la escala de diseño de simulación 0.964. La combinación de las dimensiones de la escala de informes mostró valores con p significativos (0-0,0006) y para los valores $p$ de la escala de diseño (0-0,0050). Conclusiones: la evaluación de simulación consiste en mejorar la calidad de la educación en enfermería, contribuyendo para la seguridad del paciente y al avance científico.

Descriptores: Simulación, Educación en Enfermería, Estudiantes de Enfermería, Simulación de Paciente, Desempeño de Papel.

${ }^{1}$ Curso de Enfermagem da Escola Superior em Ciências da Saúde, Brasilia, DF

${ }^{2}$ Secretaria de Saúde do Distrito Federal, Brasilia, DF

${ }^{3}$ Universidade de Brasilia, DF

${ }^{4}$ Fundação Universidade Aberta do Distrito Federal, Brasilia, DF

Autor Correspondente: Fabiola Lopes Rodrigues - Email: fabiolla.lopes02@gmail.com 


\section{INTRODUÇÃO}

São muitas as possibilidades para utilização de metodologias ativas no processo ensino-aprendizagem e, na enfermagem, essas práticas pedagógicas vêm ganhado destaque, permitindo ao estudante o protagonismo e ampliando sua responsabilidade em relação à sua formação(1-2)

As metodologias ativas utilizando a simulação realística representa uma dessas abordagens ao promover o desenvolvimento de competências em ambiente protegido, onde estudantes têm a oportunidade de testar seus conhecimentos vivenciando casos clínicos previamente simulados(3). Sendo possivel avaliar desempenho e satisfação do estudante por meio de escalas validadas, como por exemplo, escala de experiência com o debriefing e escala de design da simulação(4-5). Para a realização da simulação deve-se utilizar o briefing que consiste em orientações e informações disponibilizadas aos participantes antes da experiência da simulação e o debriefing, etapa que ocorre após a experiência da simulação(3,6).

Desse modo, os estudos conduzidos no contexto educacional sobre simulação ainda possuem lacunas não totalmente esclarecidas, sobretudo, no Brasil, justificando- se a relevância da realização deste estudo, e norteando-se pela seguinte questão: Qual a avaliação do processo ensino-aprendizagem no ambiente de simulação realística na percepção de estudantes de graduação em enfermagem? Objetivou-se avaliar o processo ensino-aprendizagem no ambiente de simulação realística na percepção de estudantes de graduação em enfermagem.

\section{METODOLOGIA}

\section{Tipo de estudo}

Trata-se de estudo transversal descritivo.

\section{Participantes da pesquisa}

A população do estudo foi composta por 64 estudantes do Curso de Graduação em Enfermagem. Os critérios de inclusão foram: estudantes que estiveram regularmente matriculados no terceiro ano do curso de enfermagem e usaram o laboratório de simulação como estratégia de ensino-aprendizagem. Foram excluídos cinco estudantes que preencheram os instrumentos de coleta de dados de forma incorreta.

\section{Local do estudo}

O cenário utilizado foi uma Instituição de Ensino Superior vinculada à Secretaria de Estado de Saúde do Distrito Federal (SES/DF).

\section{Coleta dos dados}

A coleta de dados ocorreu entre junho e setembro de 2017. Os estudantes foram divididos em quatro grupos com dezesseis participantes cada.

Planejaram as sessões de simulação, 23 docentes que utilizaram um roteiro previamente construido a partir do eixo teórico-prático e do estudo das escalas debriefing e design de simulação.

Foram realizadas quatro sessões de simulação que versaram sobre a temática da saúde do adulto e envolveram situações clínicas de terminalidade imitando o cenário hospitalar. Um manequim adulto com painel eletrônico específico para atividade simulada da marca SimulaCare Nacional foi utilizado nas sessões.

Após as sessões, foram aplicadas duas escalas, validadas para a língua portuguesa: a escala de experiência com o debriefing" e a escala de design da simulação(4-5).

A escala de experiência com o debriefing contém quatro dimensões: Analisando os pensamentos e sentimentos; Aprendendo e fazendo conexões; Habilidade do professor em conduzir o debriefing; e Orientação apropriada do professor, com um total de 20 itens.

A escala de design de Simulação" contém cinco dimensões: Objetivos e informações; Apoio; Resolução de problemas; Feedback/reflexão; e Realismo, também envolvendo 20 itens

As escalas são do tipo Likert, onde os números de "um" a "cinco" representaram o grau de concordância dos estudantes, sendo o número "um", concordância mínima e o número "cinco", concordância máxima, os quais, $1=$ discordo totalmente da afirmação; 2=discordo da afirmação; $3=$ nem concordo e nem discordo da afirmação; 4=concordo com a afirmação; e 5=concordo totalmente com a afirmação.

Assim, após as sessões de simulação, os estudantes avaliaram o processo de ensino-aprendizagem utilizando as escalas.

\section{Procedimentos de análise dos dados}

Para a análise descritiva, considerou-se o nível de concordância a partir de $60 \%$ entre os estudantes, como satisfatório para o somatório das classificações da escala Likert: 4=Concordo com a afirmação; e 5=concordo totalmente com a afirmação. Recomenda-se 50\% a $80 \%$ de consenso/concordância pelos participantes ${ }^{(7)}$

Para o tratamento dos dados utilizaram-se tabelas, frequências, porcentagens por meio da estatística descritiva e analítica univariada com associações pelo teste Qui-Quadrado considerando nível de significância $(p<0,05)$. Avaliou-se consistência interna da escala pelo Alpha de Cronbach 
considerando intervalo ideal de valores entre 0,7 e 0,9 com probabilidade de erro em $5 \%{ }^{(8)}$, a fim de verificar se todos os itens do domínio mediram a mesma característica, por meio da correlação entre as respostas em cada escala e a partir da análise das respostas dadas pelos estudantes.

\section{Procedimentos éticos}

Cumpriu-se os aspectos éticos da Resolução 466/12 do Conselho Nacional de Saúde, os participantes foram esclarecidos sobre a existência de riscos e benefícios resultantes da pesquisa aprovada pelo Comitê de Ética em Pesquisa da Fundação de Ensino e Pesquisa em Ciências da Saúde com parecer 2.050.965.

\section{RESULTADOS}

Para a aplicação das duas escalas, obteve-se o somatório das proporções referentes aos níveis de concordância mais elevados da escala likert entre os estudantes que avaliaram os aspectos relacionados ao processo ensino-aprendizagem (tabela 1).

Tabela 1 - Distribuição das respostas dos estudantes da escala de experiência com debriefing. ( $n=59)$. Brasília, DF, 2017

*Fator 1: Analisando os pensamentos e sentimentos

$\begin{array}{lccccc}\text { Itens } & \dagger 1(f \%) & +2(f \%) & \dagger 3(f \%) & +4(f \%) & \dagger 5(f \%) \\ \begin{array}{l}\text { l. O debriefing me } \\ \begin{array}{l}\text { ajudou a analisar } \\ \text { meus pensamentos. }\end{array}\end{array} & 3 / 5.0 & 11 / 18.6 & 7 / 11.8 & 11 / 18.5 & 26 / 44.0 \\ & & & & & \end{array}$

2.O facilitador re-

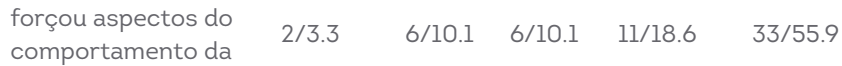
equipe.

\begin{tabular}{|c|c|c|c|c|}
\hline $\begin{array}{l}\text { 3. O ambiente de } \\
\text { debriefing foi fisica- } \\
\text { mente confortável. }\end{array}$ & $3 / 5.0$ & $5 / 8.4$ & $9 / 15.2$ & $13 / 22.0$ \\
\hline
\end{tabular}

4.Sentimentos

$\begin{array}{llllll}\text { incorretos foram } & 3 / 5.1 & 3 / 5.17 & 8 / 13.7 & 15 / 25.8 & 27 / 46.5\end{array}$

resolvidos através do

debriefing.

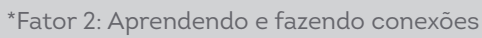

5. O debriefing ajudou-me a fazer conexões na minha $35.0 \quad 4 / 6.7 \quad 8 / 13.5 \quad 15 / 25.4 \quad 26 / 44.9$ aprendizagem.

06. O debriefing foi útil para processar a experiência de simulação.
7. O debriefing proporcionou-me oportunidades de $1 / 1.6$ $1 / 1.69$ $1 / 1.69$ aprendizagem.

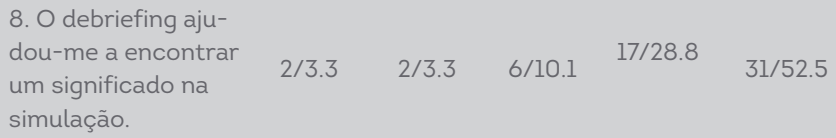

\section{As minhas dúvidas} da simulação foram respondidas pelo $2 / 3.3$ $4 / 6.78 \quad 4 / 6.78 \quad 19 / 32.2$ debriefing.
10. Tornei-me mais consciente de mim mesmo durante a sessão de debriefing.

11. O debriefing aju-

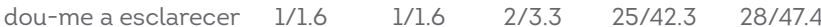
problemas.
12. O debriefing ajudou-me a fazer conexões entre teoria e situações da vida real.

*Fator 3: Habilidade do professor em conduzir o debriefing

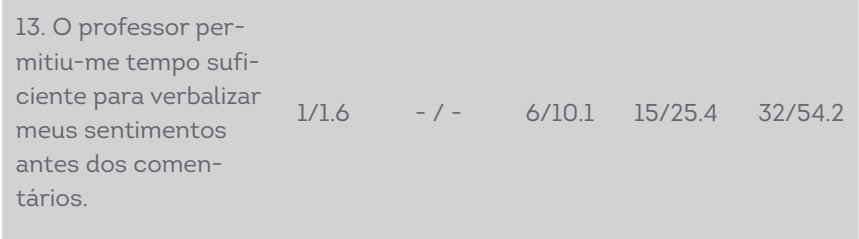

14. Na sessão de

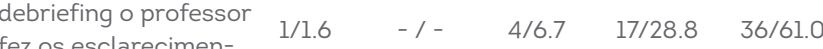
fez os esclarecimentos corretos.

\section{O debriefing} forneceu um meio $\begin{array}{llllll}\text { para eu refletir sobre } \quad 2 / 3.3 \quad-/ & 2 / 3.3 \quad 20 / 33.9 & 33 / 55.9\end{array}$ minhas ações durante a simulação.

16. Eu tive tempo $\begin{array}{llllll}\text { suficiente para escla- }^{-} & 1 / 1.6 & 4 / 6.7 & 9 / 15.2 & 15 / 25.4 & 27 / 45.7\end{array}$ recer meus questionamentos.

17. Na sessão de debriefing o professor $\begin{array}{llllll}\text { foi um especialista na } & 3 / 5.0 & 1 / 1.6 & 7 / 11.8 & 14 / 23.7 & 31 / 52.5\end{array}$ temática desenvolvida na simulação. 
${ }^{\star}$ Fator 4: Orientação apropriada do professor

18. O professor en-
$\begin{aligned} & \text { sinou a quantidade } \\ & \text { certa durante a ses- }\end{aligned}$
são de debriefing.

19. O professor realizou uma avaliação construtiva da simulação durante o

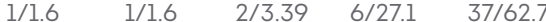
debriefing.

20. O professor forneceu orientação adequada durante o $1 / 1.6$ $1 / 1.6$ $3 / 5.08$ $14 / 23.7$ $39 / 66.1$ debriefing.

Nota: *Os fatores de 1 ao 4 representam os domínios da escala; †Os números de 1 ao 5 significam o nível de concordância mínima a máxima da escala Likert, sendo o (f\%), a frequência e porcentual das respostas obtidas.

A "Escala de experiência com debriefing" revelou em relação ao domínio (fatorl): "Analisando os pensamentos e sentimentos" que os estudantes concordaram com as afirmações, conforme os itens e seus respectivos consensos: O debriefing me ajudou a analisar meus pensamentos (62\%); O ambiente de debriefing foi fisicamente confortável (69.4\%) e; os Sentimentos incorretos foram resolvidos através do debriefing (72\%).

Referente ao domínio (fator 2): “Aprendendo e fazendo conexões", constataram-se os seguintes percentuais de concordância: O debriefing ajudou-me a fazer conexões na minha aprendizagem (70.3\%); O debriefing foi útil para processar a experiência de simulação (91.5\%); O debriefing proporcionou-me oportunidades de aprendizagem (93\%); O debriefing ajudou-me a encontrar um significado na simulação (81\%); As minhas dúvidas da simulação foram respondidas pelo debriefing (80\%); Tornei-me mais consciente de mim mesmo durante a sessão de debriefing (78\%); e 0 debriefing ajudou-me a fazer conexões entre teoria e situações da vida real (91\%).

Com relação ao domínio (fator 3): "Habilidade do professor em conduzir o debriefing", os estudantes avaliaram os itens com consenso elevado em todas as afirmações, as quais: O professor permitiu-me tempo suficiente para verbalizar meus sentimentos antes dos comentários (80\%); 0 debriefing forneceu um meio para eu refletir sobre minhas ações durante a simulação (90\%); Eu tive tempo suficiente para esclarecer meus questionamentos (71\%) e; Na sessão de debriefing o professor foi um especialista na temática desenvolvida na simulação (76\%).
Para o domínio (fator 4): "Orientação apropriada do professor", verificou-se que os estudantes concordaram ou concordaram totalmente com as afirmações: O professor ensinou a quantidade certa durante a sessão de debriefing (73\%); O professor realizou uma avaliação construtiva da simulação durante o debriefing (90\%); O professor forneceu orientação adequada durante o debriefing (90\%).

Em seguida, os estudantes avaliaram as sessões utilizando a escala do design de simulação com itens distribuídos entre os domínios ou fatores (tabela 2).

Tabela 2 - Distribuição das respostas dos estudantes da escala de design da simulação. (N=59), Brasília, DF, 2017

\begin{tabular}{|c|c|c|c|c|c|}
\hline Itens & $+1(f \%)$ & $+2(f \%)$ & †3(f \%) & $+4(\mathrm{f} \%)$ & $+5(f \%)$ \\
\hline $\begin{array}{l}\text { 1.No inicio da simu- } \\
\text { lação foi fornecida } \\
\text { informação suficiente } \\
\text { para proporcionar } \\
\text { orientação e incen- } \\
\text { tivo. }\end{array}$ & $3 / 5.0$ & $11 / 18.6$ & $7 / 11.8$ & $11 / 18.5$ & $26 / 44.0$ \\
\hline
\end{tabular}

2. Eu entendi clara-

mente a finalidade e os objetivos da simu-

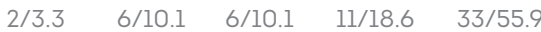
lação.

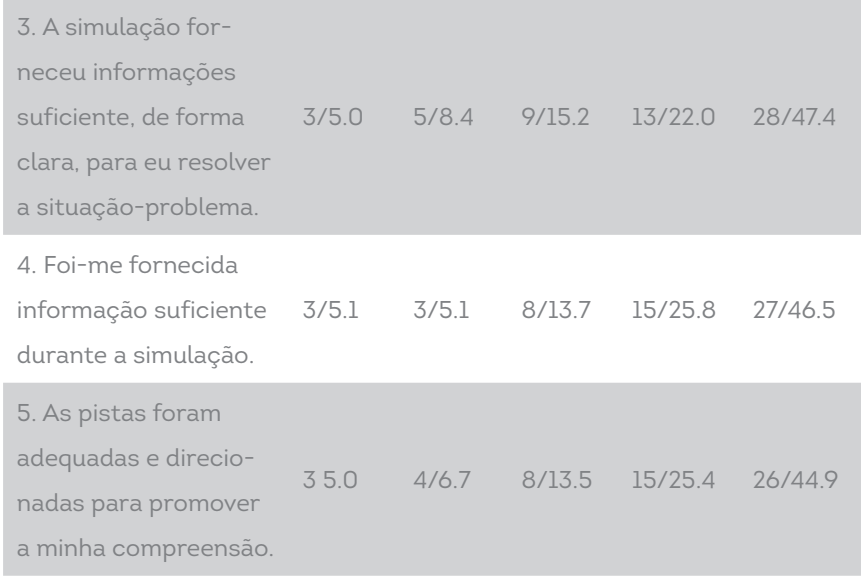

${ }^{\star}$ Fator 2: Apoio

6. O apoio foi ofere-
cido em tempo opor- $1 / 1.6 \quad-/-\quad 3 / 5.0 \quad 19 / 32.2 \quad 35 / 59.3$
tuno.

7. A minha necessida-

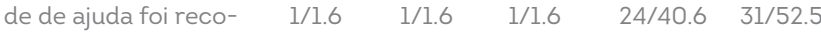

nhecida.

8. Eu senti-me apoia-

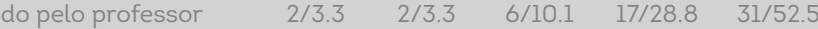

durante a simulação. 


\begin{tabular}{|c|c|c|c|c|c|}
\hline $\begin{array}{l}\text { processo de aprendi- } \\
\text { zagem. }\end{array}$ & $2 / 3.3$ & $4 / 6.7$ & $4 / 6.7$ & $19 / 32.2$ & $28 / 47.4$ \\
\hline \multicolumn{6}{|c|}{ `Fator 3: Resolução de problemas } \\
\hline $\begin{array}{l}\text { 10. A resolução de } \\
\text { problemas de forma } \\
\text { autônoma foi facili- } \\
\text { tada. }\end{array}$ & $4 / 6.7$ & $1 / 1.6$ & $5 / 8.4$ & $17 / 28.8$ & $29 / 49.1$ \\
\hline $\begin{array}{l}\text { 11. Fui incentivado } \\
\text { a explorar todas as } \\
\text { possibilidades da } \\
\text { simulação. }\end{array}$ & $1 / 1.6$ & $1 / 1.6$ & $2 / 3.3$ & $25 / 42.3$ & $28 / 47.4$ \\
\hline
\end{tabular}

12. A simulação foi projetada para o meu

nivel especifico de $\quad 1 / 1.6 \quad-/-\quad 3 / 5.0 \quad 22 / 37.2 \quad 32 / 54.2$ conhecimento e habi-

lidades.

13. A simulação permi-
tiu-me a oportunidade
de priorizar as avalia-
ções e os cuidados de

14. A simulação pro-

porcionou-me uma

$\begin{array}{llllll}\text { oportunidade de esta- } & 1 / 1.6 & -/ & -4 / 6.7 & 17 / 28.8 & 36 / 61.0\end{array}$

belecer objetivos para

o meu paciente.

\begin{tabular}{|c|c|c|c|c|c|}
\hline $\begin{array}{l}\text { 15. O feedback forne- } \\
\text { cido foi construtivo. }\end{array}$ & $2 / 3.3$ & $-1-$ & $2 / 3.3$ & $20 / 33.9$ & $33 / 55.9$ \\
\hline 16. O feedback foi & & & & & \\
\hline $\begin{array}{l}\text { fornecido em tempo } \\
\text { oportuno. }\end{array}$ & $1 / 1.6$ & $4 / 6.7$ & $9 / 15.2$ & $15 / 25.4$ & $27 / 45.7$ \\
\hline
\end{tabular}

17. A simulação permi-

tiu-me analisar meu

próprio comporta-

$\begin{array}{lllll}3 / 5.0 & 1 / 1.6 & 7 / 11.8 & 14 / 23.7 & 31 / 52.5\end{array}$

mento e ações.

$\begin{aligned} & \text { 18. Após a simulação } \\ & \text { houve oportunidade } \\ & \text { para obter orienta- } \\ & \text { ção/feedback do pro- } \\ & \text { fessor, a fim de cons- }\end{aligned}$
truir conhecimento
para outro nivel.

*Fator 5: Realismo

19. O cenário se asse-
melhava a uma situa-
ção da vida real.

20. Fatores, situações

e variáveis da vida real

$\begin{array}{llllll}\text { foram incorporados } & 1 / 1.6 & 1 / 1.6 & 3 / 5.0 & 14 / 23.7 & 39 / 66.1\end{array}$ ao cenário de simu-

lação.

Nota: ${ }^{*}$ s fatores de 1 ao 4 representam os domínios da escala; †Os números de 1 ao 5 significam o nível de concordância mínima a máxima da escala Likert, sendo (f\%), a frequência e percentual das respostas obtidas.

Ao considerar o domínio (fator 1): "Objetivos e informações", verificou-se as seguintes avaliações e seus respectivos consensos: No início da simulação foi fornecida informação suficiente para proporcionar orientação e incentivo (62.5\%); Eu entendi claramente a finalidade e os objetivos da simulação (74.5\%); A simulação forneceu informação suficiente, de forma clara, para eu resolver a situação-problema (70\%); e As pistas foram adequadas e direcionadas para promover a minha compreensão (70\%).

Conforme o domínio (fator 2): “Apoio", os estudantes avaliaram positivamente as afirmações: $O$ apoio foi oferecido em tempo oportuno (91.5\%); A minha necessidade de ajuda foi reconhecida (93\%); Eu me senti apoiado pelo professor durante a simulação (81\%).

Sobre o domínio (fator 3): "Resolução de problemas", os estudantes avaliaram assertivamente os itens com os respectivos níveis de concordância: A resolução de problemas de forma autônoma foi facilitada (78\%); A simulação foi projetada para o meu nível específico de conhecimento e habilidades (91\%); A simulação permitiu-me a oportunidade de priorizar as avaliações e os cuidados de enfermagem (80\%); e A simulação proporcionou-me uma oportunidade de estabelecer objetivos para o meu paciente (90\%).

Em relação ao domínio (fator 4): "Feedback/Reflexão", os estudantes avaliaram com os seguintes consensos: $O$ feedback fornecido foi construtivo (90\%); O feedback foi fornecido em tempo oportuno (71\%); A simulação permitiu-me analisar meu próprio comportamento e ações (76\%); Após a simulação houve oportunidade para obter orientação/feedback do professor a fim de construir conhecimento para outro nivel (73\%).

Referente ao domínio (fator 5): "Realismo”, os estudantes avaliaram assertivamente os itens: O cenário se assemelhava a uma situação da vida real (90\%); e Fatores, situações e variáveis da vida real foram incorporados ao cenário de simulação (90\%).

Verificando-se a confiabilidade da escala e sua consistência interna, bem como, sua reprodutibilidade com base 
nas respostas dos estudantes, o Alpha de Cronbach foi utilizado. A confiabilidade interna da escala de experiência com debriefing foi de 0.972 e da escala do design de simulação foi de 0.964 .

Utilizou-se o teste Qui-quadrado para verificar diferenças significativas das associações entre os quatro domínios de análise da escala de debriefing, obtiveram-se valores significativos que variaram de $(0-0,0006)$ e para a escala de design, pelas associações entre os cinco domínios, verificaram-se valores de (0-0,0050) (tabelas 3 e 4).

Tabela 3. Associações das avaliações entre os domínios da escala de debriefing. ( $N=59)$, Brasília, DF, 2017

\begin{tabular}{lll}
\hline $\begin{array}{l}\text { *Relação entre } \\
\text { os dominios }\end{array}$ & $\dagger \chi^{2}$ & $\neq$-valor \\
\hline Fatorl X Fator2 & 0.5121436 & 0.0000 \\
\hline Fatorl X Fator3 & 0.4327901 & 0.0006 \\
\hline Fatorl X Fator4 & 0.4381063 & 0.0005 \\
\hline Fator2 X Fator3 & 0.4652727 & 0.0002 \\
\hline Fator2 X Fator4 & 0.4796788 & 0.0001 \\
\hline Fator3 X Fator4 & 0.6546508 & 0.0000 \\
\hline
\end{tabular}

Nota: *Fator 1: Analisando os pensamentos e sentimentos; Fator 2: Aprendendo e fazendo conexões; Fator 3: Habilidade do professor em conduzir o debriefing; Fator 4: Orientação apropriada do professor. †Teste Qui-quadrado; łsignificância.

Tabela 4. Associações das avaliações entre os domínios da escala de design. (N=59), Brasília, DF, 2018.

\begin{tabular}{|lll}
\hline $\begin{array}{l}\text { Relação entre } \\
\text { os dominios }\end{array}$ & Teste Qui-quadrado & $p$-valor \\
\hline Fatorl X Fator2 & 0.5115748 & 0.0000 \\
\hline Fator1 X Fator3 & 0.5922412 & 0.0000 \\
\hline Fator1 X Fator4 & 0.4527091 & 0.0004 \\
\hline Fator1 X Fator5 & 0.5028623 & 0.0000 \\
\hline Fator2 X Fator3 & 0.6113865 & 0.0000 \\
\hline Fator2 X Fator4 & 0.4925636 & 0.0000 \\
\hline Fator2 X Fator5 & 0.3898106 & 0.0024 \\
\hline Fator3 X Fator4 & 0.5717642 & 0.0000 \\
\hline Fator3 X Fator5 & 0.3632037 & 0.0050 \\
\hline Fator4 X Fator5 & 0.3969829 & 0.0022 \\
\hline
\end{tabular}

Nota: *Fator 1: Objetivos e informações; Fator 2: Apoio; Fator 3: Resolução de problemas Fator 4: Feedback/Reflexão Fator 5: Realismo. †Teste Qui-quadrado; łsignificância.

\section{DISCUSSÃO}

A avaliação do processo ensino-aprendizagem no ambiente de simulação, na percepção dos estudantes, foi satisfatória e de modo homogênea. As escalas de debriefing e do design apontaram niveis de concordância elevados, como recomendado por autores(7).

Neste estudo, o debriefing permitiu aos estudantes trabalhar o desenvolvimento de competências e a refletir sobre os cenários simulados e suas ações aplicadas para solucionar os objetivos, desenvolvendo a comunicação, as habilidades técnicas e a atitude profissional perante situações emergenciais. O treino simulado possibilita essa oportunidade sem risco de dano ao paciente, e a aprendizagem adquirida possibilita vivenciar e solucionar, com maior segurança, situações críticas(9-10).

A avaliação dos estudantes sobre a análise de pensamentos e sentimentos no debriefing foi satisfatória, porém, para alguns estudantes, questões emocionais vieram à tona, como sentimentos de medo, ansiedade e relacionamento com seus pares. Autores(11), relatam que os principais fatores estressores dos estudantes na simulação estão relacionados com a falta de competência e dificuldades de relacionamento. Dessa forma, serve de subsidio para que os docentes de enfermagem elaborem estratégias e cenários que permitam a minimização de fatores estressores observados.

O planejamento exige uma árdua preparação do docente, pois mesmo havendo uma boa organização, falhas podem acontecer e, caso não sejam esclarecidas, podem comprometer o sucesso da estratégia(12). As escalas de design e de debriefing contribuíram para elaboração do roteiro de simulação pelos docentes que construíram as situações e prepararam o cenário por meio do aprofundamento teórico-prático e a partir dos itens que seriam avaliados pelos estudantes, reduzindo as chances de falhas no processo.

$\mathrm{Na}$ simulação, os educadores devem se concentrar não apenas nos domínios psicomotores, mas também em reconhecer a influência dos domínios afetivos e cognitivos(13). Destaca-se a necessidade de investir na construção de cenários com design detalhado para que sejam os mais realísticos possíveis baseados em fatos do cotidiano, bem como, no preparo dos facilitadores ou docentes para que possam realizar um debriefing problematizador(14).

Os valores Alpha de Cronbach das duas escalas foram elevados, haja vista que as duas mediram consistentemente as características para a quais foram empregadas(8). Do mesmo modo, os resultados dos $p$-valores exibiram diferenças significativas(15) dentro de uma margem de erro de $5 \%$, inferindo-se a importância de cada um dos domínios das escalas. 


\section{CONTRIBUIÇÕES PARAA PRÁTICA}

A contribuição desse estudo pauta-se na utilização de escalas validadas de avaliação da simulação, o que permite que docentes e estudantes acompanhem os resultados da simulação por meio de instrumentos confiáveis e reflitam nos pontos em que podem corrigir e aprimorar as suas vivências em ambientes simulados de aprendizagem.

\section{LIMITAÇÕES DO ESTUDO}

O estudo apresenta como limitação a inclusão de estudantes de uma única Instituição de Ensino Superior, impossibilitando uma análise ampliada, o que torna necessário a sua replicação em outras instituições de ensino. No entanto, destacam-se os benefícios advindos da avaliação da prática profissional em cenários simulados.

\section{REFERÊNCIAS}

1. Marques LMNSR. Active methodologies as strategies to develop education in values in nursing graduation. Anna Nery Rev. Enferm. 2018;22(3):e20180023. doi: http://dx.doi.org/10.1590/180757622014.1177

2. Freitas DA, Santos EMS, Lima LVS. Teachers' knowledge about teaching-learning process and its importance for professional education in health. Interface - Comunic. Saude. Educ. 2016:20(57): 43748. doi: http://dx.doi.org/10.1590/1807-57622014.1177

3. Barreto DG, Silva KGND, Moreira SSCR, Silva TSD, Magro MCDS. Realistic simulation as a teaching strategy: an integrative review. Rev. Baiana Enferm. [internet] 2014 [cited May 07, 2019]:28(2):208-214. Available from: https://portalseer.ufba.br/index.php/enfermagem/ article/viewFile/8476/8874

4. Almeida RGS, Mazzo A, Martins JCA, Coutinho VRD, Jorge BM, Mendes IAC. Validation to Portuguese of the Debriefing Experience Scale. Rev. Bras Enferm. 2016; 69(4):658-64. doi: http://dx.doi.org/10.1590/0034$7167.2016690413 i$

5. Almeida RGS, Mazzo A, Martins JCA, Pedersoli CE, Fumincelli L, Mendes IAC. Validation for the portuguese language of the simulation design scale. Texto Contexto Enferm. 2015;23(6)1007-13. doi: http:// dx.doi.org/10.1590/0104-0707201500004570014

6. Ferreira RP, Guedes HM, Oliveira DWD, Miranda JL. Realistic simulation as a method of teaching in the learning of the health field students. R. Enferm. Cent. O. Min. 2018:8:e2508. doi: http://dx.doi.org/10.19175/ recom.v7i0.2508

7. Scarparo AF, Laus AM, Azevedo ALCS, Freitas MRI, Gabriel CS, Chaves LDP. Reflections on the use of Delphi technique in research in nursing. Rev Rene. [internet]. 2012 [cited May 11, 2019];13(1):242-51. Available from: http://www.periodicos.ufc.br/rene/ article/view/3803/3000. 8.Taber, K.S. The Use of Cronbach's Alpha When Developing and

\section{CONCLUSÃO}

Os resultados demonstraram que a experiência da simulação realística enquanto processo ensino-aprendizagem foi avaliado de forma satisfatória pelos estudantes alcançando níveis de concordância elevados e significância estatística a partir dos achados analíticos, confirmando assim a simulação como estratégia capaz de agregar valor à educação superior em enfermagem.

Contribuições dos autores: FLRG, LMM, LMMB, MCM, GSA, FCVF, contribuíram para a concepção e desenho do estudo, análise e interpretação dos dados, redação do artigo, revisão crítica e revisão final.
Reporting Research Instruments in Science Education. Res Sci Educ. 2018:48:1273-1296. doi: https://doi.org/10.1007/s11165-016-9602-2

9. Presado MHCV, Colaço S, Rafael H, Baixinho CL, Félix I, Saraiva C. Rebelo I. Learning with High Fidelity Simulation. Ciênc. saúde coletiva. 2018:23(1):51-59. doi: http://dx.doi.org/10.1590/141381232018231.23072017

10. Mesquita HCT, Santana BS, Magro MCS. Effect of realistic simulation combined to theory on self-confidence and satisfaction of nursing professionals. Anna Nery Rev. Enferm. 2019;23(1). doi: http://dx.doi. org/10.1590/2177-9465-EAN-2018-0270

11. Boostel R, Felix JVC, Bortolato-Major C, Pedrolo E, Vayego SA. Mantovani MF. Stress of nursing students in clinical simulation: a randomized clinical trial. Rev. Bras. Enferm. 2018;71(3):967-974. doi: http://dx.doi.org/10.1590/0034-7167-2017-0187

12. Miranda FBG, Mazzo A, Pereira GAJ. Use of high fidelity simulation in the preparation of nurses for urgency and emergency care: scoping review. Sci Med. 2018;28(1):28675. doi: http://dx.doi.org/10.15448/19806108.2018 .1 .28675

13. Al Sabei S D. Lasater K. Simulation debriefing for clinical judgment development: A concept analysis. Nurse Educ Today. 2016:45:42-7. doi: http://dx.doi.org/10.1016/j.nedt.2016.06.008

14. Cogo ALP, Lopes EFS, Perdomini FRI, Flores GE, Santos MRR. Building and developing realistic simulation scenarios on safe drug administration. Rev Gaúcha Enferm. 2019; 40(esp):e20180175. doi: https://doi.org/10.1590/1983-1447.2019.20180175

15. Ferreira JC, Patino CM. What does the $p$ value really mean? J Bras Pneumol. 2015:41(5):485. doi: http://dx.doi.org/10.1590/S180637132015000000215 